\title{
Hydrothermal Synthesis and Characterizations of MOF-199 Using Renewable Template
}

\author{
N. Mahadi ${ }^{1, a}$, H. Misran ${ }^{2, b}$, S. Z. Othman ${ }^{3, c}$, N. S. Jamaludin, A. Manap, \\ and N. F. S. Anuar \\ 1,2,3 Nanoarchitectonic Laboratory, UNITEN Putrajaya Campus, \\ College of Engineering, Jalan Ikram-UNITEN, 43000 Kajang, Selangor, MALAYSIA.

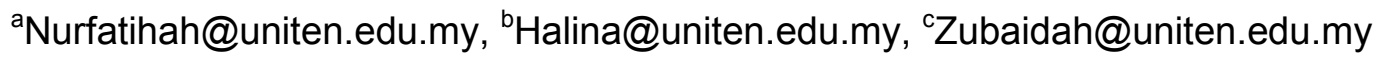

\begin{abstract}
Keywords: Microporous, metal-organic framework, MOF-199, renewable template, fatty alcohol, adsorption isotherms
\end{abstract}

\begin{abstract}
A three-dimensional (3D) copper-based metal-organic frameworks or MOF-199 were successfully synthesized by utilizing palm oil based fatty alcohols that acted as renewable template. The synthesis of MOF-199 employed different length of straight chain of fatty alcohols namely octyl, decyl, lauryl, myristyl and cetyl alcohols derived from palm oil using similar method to those reported earlier with some modifications. The microstructure of MOF-199 exhibited single particles with fine octahedral morphology at various particle sizes in the range of ca. 15-60 $\mu \mathrm{m}$. The nitrogen adsorption isotherms were conducted to estimate the surface area and pore volume of the materials and were at ca. $400-1100 \mathrm{~m}^{2} \mathrm{~g}^{-1}$ and $0.17-0.43 \mathrm{~cm}^{3} \mathrm{~g}^{-1}$ respectively. The surface area and pore volume of the materials decreased with longer straight chain fatty alcohol possibility due to mobility of the long carbon chain.
\end{abstract}

\section{Introduction}

As a new class in the family of microporous materials, metal-organic frameworks (MOFs) were considered as a versatile nanoporous materials with flexible structures [1]. MOFs were firstly invented by O. Yaghi et al. in 1990's [2]. These materials became an excellent candidate for various application including hydrogen and other gases storage, gas purification, catalyst and drug delivery as it has the ability to store the guest molecules inside porous structure [1-4].

Generally, MOFs composed of metal cluster ions linked by inorganic linker building blocks. The organic and inorganic building blocks constructed in multidentate repetition entities to form 3D MOFs to develop a network of channels or pores inside the structure. The topology of MOFs has larger variability where these materials usually rely on the types of metal ions and linkers used to formed unique geometrical properties [3]. MOFs materials especially MOF-199 is one of the most studied as it has large surface area, high porosity, low density, good thermal stability, high chemical stability and stable and have high affinity towards water molecules $[3,4]$.

Since their discoveries, MOF-199 was synthesized from various routes to optimize the production yielded and enhance of well-defined single particle structure. The properties of this microporous material were highly depending on the synthesis method, reaction temperature, ratio of organic to inorganic materials and amount of solvents used [5,6]. The addition of fatty alcohols in this study was similar to the conventional hydrothermal method after preparing the solution with some modification. The presence of straight chain fatty alcohols helped the organic linker to crosslink with inorganic metal cluster resulting in fine single particle morphology.

\section{Experimental.}

Materials. All chemicals were used as received. Copper (II) nitrate trihydrate (99.5\%, $\left.\mathrm{Cu}\left(\mathrm{NO}_{3}\right)_{2} .3 \mathrm{H}_{2} \mathrm{O}\right)$, ethanol $\left(99.8 \%, \mathrm{C}_{5} \mathrm{H}_{5} \mathrm{OH}\right)$ 1,3,5-benzene tricarboxylic acid (BTC acid, $\mathrm{C}_{9} \mathrm{H}_{6} \mathrm{O}_{6}$ ) were purchased from Merck. Fatty alcohols including octyl (C8), decyl (C10), lauryl (C12), myristyl (C14) and cetyl (C16) alcohols were purchased from Emery Oleochemical (M) Sdn Bhd. 
Synthesis Method. The method in this study was similar to Misran et al. and Wang et al. with some modifications [1,7-9]. The materials manipulated in the experiment generally referred to Chowdury et al. [10] with some modification. The synthesis of MOF-199 made up of two solutions. The first solution comprised of ethanol $\left(\mathrm{C}_{2} \mathrm{H}_{5} \mathrm{OH}, 0.34 \mathrm{~mol}\right)$ and 1,3,5-benzene tricarboxylic acid (BTC acid, 0.009 mol). The second solution contained copper (II) nitrate trihydrate $\left(\mathrm{Cu}\left(\mathrm{NO}_{3}\right)_{2} .3 \mathrm{H}_{2} \mathrm{O}, 0.02 \mathrm{~mol}\right)$ and distilled water $\left(\mathrm{H}_{2} \mathrm{O}, 0.56 \mathrm{~mol}\right)$. Both solutions were added together while stirring until both were completely dissolved at room temperature. Then, an amount of fatty alcohols were added into the solution and stirred for several minutes until completely dissolved. The addition of myristyl (C14) and cetyl (16) alcohol were exposed to heat to melt their solid form before being transferred in the solution. The resulting solutions were transferred into a Teflon-lined autoclave. It was heated overnight at $413 \mathrm{~K}$ before being cooled to room temperature and washed using distilled water. It was found that longer carbon chain in fatty alcohol needed more repetition to wash the sample. Next, the precipitate was dried for few hours to form dark blue powder.

Characterizations. The synthesized samples prepared in this study were subjected to X-Ray Diffraction machine using Shimadzu XRD-6000 with $\mathrm{CuK} \alpha$ radiation for $2 \theta=5^{\circ}-45^{\circ}$. The parameters used were $30 \mathrm{kV}$, scan speed $2^{\circ} / \mathrm{min}$ and the scan step applied was $0.02^{\circ}[7,8]$. The morphologies of the synthesized MOF-199 were obtained using Phenom G2 Pro Scanning Electron Microscope with acceleration voltage of $5 \mathrm{kV}$. The sample undergoes degassing process for 12 hours after pre-treat overnight in oven. The nitrogen adsorption-desorption analyses were done at liquid nitrogen temperature $77 \mathrm{~K}$ by Quantachrome Autosorb iQ.

\section{Results and Discussion}

MOF-199 powder with various addition of fatty alcohols (C8, C10, C14 and C16) obtained in this study were found to be dark blue powder after drying overnight to remove absorbed water. However, these materials changed to turquoise once they were exposed to environment for few seconds which suggested that the samples will undergo colour change. Those were due to absorption of water molecules or humidity from the surrounding.

X-Ray Diffraction Analyses. The XRD patterns of MOF-199 obtained in this study are shown in Fig. 1. All samples exhibited MOF-199 patterns except a sample prepared with additional lauryl alcohol (C12) during synthesis. The results shows the XRD patterns exhibited twelve prominent peaks assigned to (200), (220), (222), (400), (500), (440), (600), (551), (731), (751), (773) and (882). The highest peak was exhibited at $2 \theta=11.92^{\circ}$. The XRD patterns observed in this study were similar to those reported before suggesting that MOF-199 produces using our method were successfully synthesized. Fig. 1 shows that the addition of octyl (C8), decyl (C10) and lauryl (C16) alcohol increase the intensity of the prominent peak at (222) while the present of lauryl and myristyl alcohol ( $\mathrm{C} 12$ and $\mathrm{C} 14)$ respectively reduce the prominent peak intensity. The addition of lauryl alcohol (C12) results in different pattern as compared to other MOF-199 patterns. This suggested due to polymer-like behaviour where the longer straight carbon chains of C12 reduce the mobility in the solution to react properly and completely, therefore, the number of well-defined octahedral particles would reduce while the amorphous morphology increased at the same time. Based on the result, the preparation of MOF-199 with the addition of C12 alcohol was suggested to apply heat to the $\mathrm{C} 12$ alcohol before being added into the MOF-199 solution. Besides, the additions of fatty alcohols also enhanced the formation of single particles and improved the relative crystallinity of the structures. From C8 to $\mathrm{C} 16$ (except for $\mathrm{C} 12$ ), the peak intensity of (222) reflection plane were also increased as the carbon chain of fatty alcohol increased due to higher crystallinity of MOF-199.

Morphology Analyses. Fig. 2 shows the morphology of the as-synthesized MOF-199 in this study using Phenom G2 Pro at acceleration voltage $5 \mathrm{kV}$. The samples successfully presented many single particles with well-defined octahedral shapes at ca. $15-60 \mu \mathrm{m}$ particle sizes. The number of 
successfully formed single particles increased when fatty alcohols (C8 until C16) were added during synthesis process as compared to the sample without fatty alcohol. The SEM images of all samples with and without fatty alcohols exhibited various morphology of MOF-199. The morphology of as-synthesized MOF-199 without fatty alcohol in this study shows the microstructure of single particles with well-defined octahedral structure. The morphology of assynthesized MOF-199 samples with the presence of C8 and C10 showed well-defined single particles structure with sharp edges octahedral shape. However, the addition of $\mathrm{C} 12$ during synthesis resulted in rarely found well-defined octahedral microstructure while the amorphous structures were found dominantly from the sample. The amorphous structures have poorly defined patterns as they formed irregularly. The presence of amorphous structures was suggested due to incomplete reaction. The addition of $\mathrm{C} 14$ during synthesis produced well-defined particle structure with less sharp edges and more uniform particle sizes. The addition of $\mathrm{C} 16$ resulted in well-defined octahedral structure with some carbonaceous-like around the crystals structure.

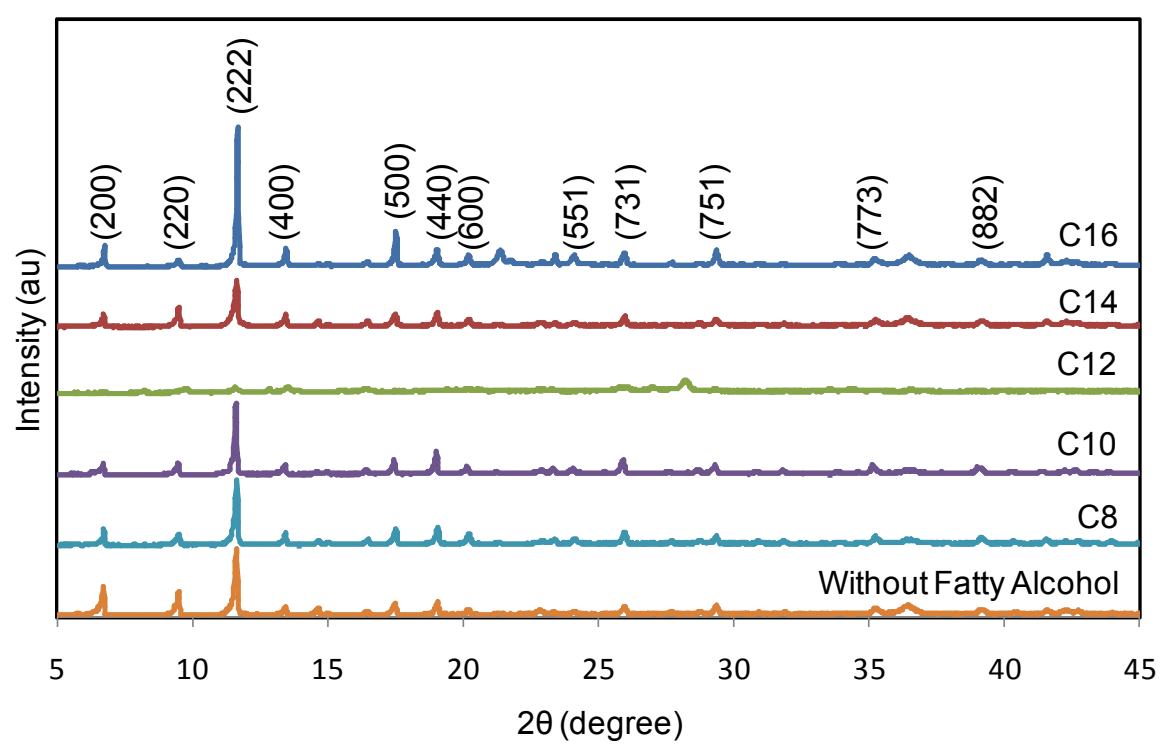

Fig. 1: The XRD patterns of as-synthesized MOF-199 with and without fatty alcohols prepared in this study.

Nitrogen Adsorption Isotherms. The adsorption-desorption isotherms of MOF-199 prepared in this study are shown in Fig. 3. The properties of these structures were determined by nitrogen $\mathrm{N}_{2}$ adsorption-desorption analysis at $77 \mathrm{~K}$ liquid nitrogen temperature. The dried MOF-199 samples (with and without fatty alcohols added) exhibited Type I isotherm suggesting the nature of the microporous materials. Hysteresis loop in the long plateau indicated relatively small amount of nitrogen $\mathrm{N}_{2}$ adsorption on the open surface which disclosed the present of permanent open cavity. However, the sorption isotherms with the addition of lauryl alcohol unsuccessfully obtained in this study somehow this sample need to undergo several study to exhibit better outcome. Meanwhile, the addition of lauryl alcohol demonstrates and follows non-porous behaviour. The BET and Langmuir surface area obtained by all sample by $\mathrm{N}_{2}$ sorption isotherm in this study are shown in Table 1. The surface area estimated using Langmuir method and BET method were nearly similar suggesting the presence of microporous and mesoporous structure. The sample prepared without the addition of fatty alcohol exhibited higher surface area due to the existence of non-defined amorphous structure as observed in SEM images and different length of straight carbon chain in fatty alcohol added in the synthesis method affected the adsorption-desorption ability by each sample. 

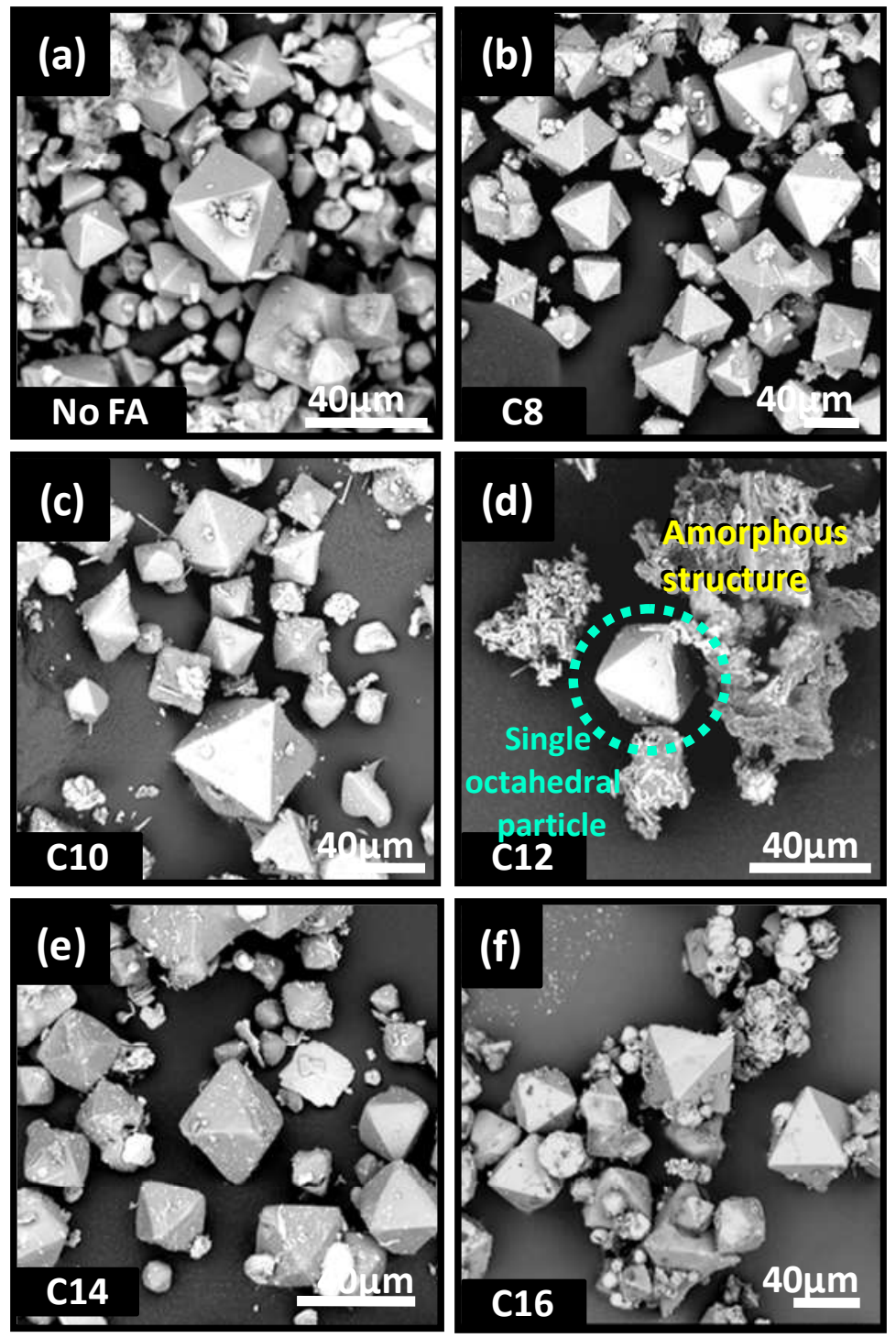

Fig. 2: The SEM images of MOF-199 produced in this study. Sample (a) synthesized without fatty alcohol while (b), (c), (d), (e) and (f) synthesized with the addition of fatty alcohols with different carbon chain.

Table 1: Porosity characteristics of MOF-199 synthesized by different length of carbon chain.

\begin{tabular}{cccc}
\hline MOF-199 & ${ }^{1} \mathrm{~S}_{\mathrm{BET}}\left(\mathrm{m}^{2} / \mathrm{g}\right)$ & ${ }^{2} \mathrm{~S}_{\text {Langmuir }}\left(\mathrm{m}^{2} / \mathrm{g}\right)$ & ${ }^{3}$ Pore Volume $\left(\mathrm{cm}^{3} / \mathrm{g}\right)$ \\
\hline Without FA (No FA) & 1104.9 & 1117.6 & 0.43 \\
C8 & 832.4 & 928.4 & 0.35 \\
C10 & 446.1 & 452.7 & 0.20 \\
C14 & 414.6 & N/A & 0.17 \\
C16 & 35.9 & 29.9 & 0.002
\end{tabular}

\footnotetext{
${ }^{*}{ }^{1}$ By BET plot, ${ }^{2}$ By Langmuir plot, ${ }^{3}$ By t-plot
} 


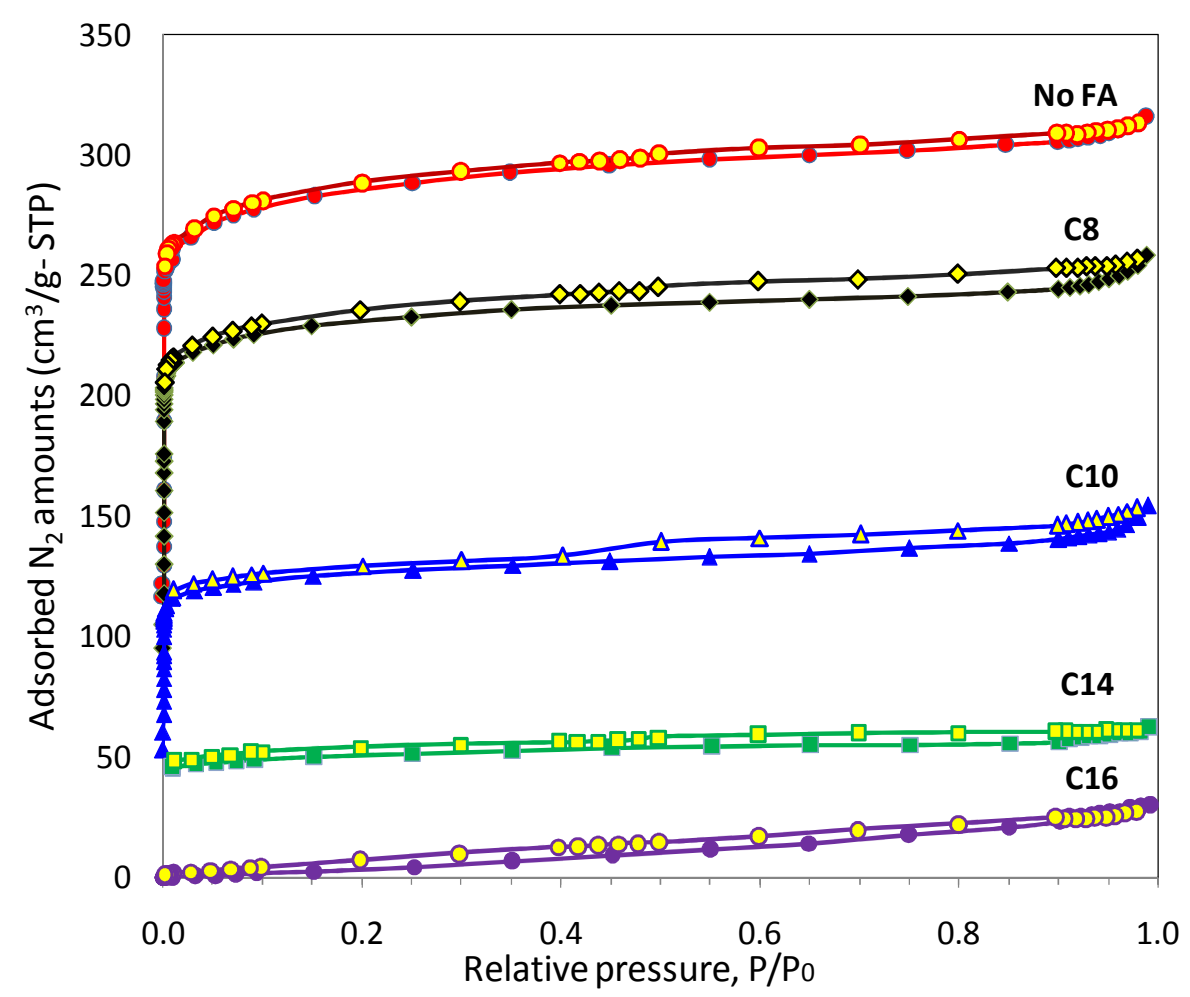

Fig. 3: The nitrogen, $\mathrm{N}_{2}$ adsorption isotherms of MOF-199 produced in this study.

\section{Conclusions}

In summary, MOF-199 with well-defined octahedral structures were successfully synthesized in this study using renewable template method by utilizing fatty alcohols with $\mathrm{C} 8-\mathrm{C} 16$ carbon chain. The addition of fatty alcohols with various lengths of carbon chains significantly improved the morphology and relative crystallinity of the materials. The presence of fatty alcohol during the synthesis increased the amount of particles yield in MOF-199. Thus, fatty alcohols were suggested to be suitable renewable template in the synthesis of MOF-199.

\section{Acknowledgements}

This work was financially supported by Ministry of Higher Education under FRGS Scheme (20130106 and 20130104) and ERGS Scheme (20130901). The autors also would like to thank Universiti Tenaga Nasional (UNITEN) for providing high class education center to accomplish this project and NanoMalaysia for their Phenom G2 Pro Scanning Electron Microscope (SEM) machine.

\section{References}

[1] H. Misran, S. Z. Othman, A. Manap, N. I. M. Pauzi and S. Ramesh, Sci Adv. Mater., 6 (7), (2014), p. 1638-1644.

[2] H. Li, Eddaoudi, M. O'Keeffe, and O. M. Yaghi, Nature 402, (1999) 276.

[3] K. S. Lin, A. K. Adhikari, C. N. Ku, C. L. Chiang, H. Kuo, Inter. J. Hydrogen Energ., Vol. 37 (18), (2012), p. 13865-13871.

[4] S. L. Serna, M. A. O. Tolentino, M. D. L. L. Nunez, A. S. Cruz, A. G. Vargas, R. C. Sierra, H. I. Beltran, J. Flores, J. Alloys Compd., Vol. 540, (2012), p. 113-120.

[5] Y. K. Seo, G. Hundal, I. T. Jang, Y. K. Hwang, C.H. Jun, J. S. Chang, Micropor. Mesopor. Mater., Vol. 119 (1-3), (2009), p. 331-337. 
[6] A. K. Cheetham, C. N. R. Rao, R. K. Feller, Chem. Commun., (2006) 4780.

[7] H. Misran, R. Singh and M. A. Yarmo, Micropor. Mesopor. Mater., 112 (1), (2008) p. 243253.

[8] S. Z. Othman, H. Misran, A. M. Aminuddin, M. A. Salim, N. N. H. Shah and N. A. A. Razak, Malays. J. Microsc., Vol. 9, (2013), p. 62-65.

[9] Q. M Wang, D. Shen, M. Bulow, M. L. Lau, S. Deng, F. R Fitch N. O. Lemccoff, J. Semanscin, Micropor. Mesopor. Mater., Vol. 55, (2002), p. 217.

[10] P. Chowdhury, C. Bikkina, D. Meisteer, F. Dreisbach, S. Gumma, Micropor. Mesopor. Mater., Vol. 117, (2009), p. 406-413. 